\title{
Stability of persistent currents in a Bose-Einstein condensate confined in a toroidal trap
}

\author{
M. Ögren ${ }^{1,2}$ and G. M. Kavoulakis ${ }^{3}$ \\ ${ }^{1}$ ARC Centre of Excellence for Quantum-Atom Optics, School of Physical Sciences, \\ University of Queensland, Brisbane, Queensland 4072, Australia \\ ${ }^{2}$ Mathematical Physics, Lund Institute of Technology, P.O. Box 118, SE-22100 Lund, Sweden \\ ${ }^{3}$ Technological Education Institute of Crete, P.O. Box 1939, GR-71004, Heraklion, Greece
}

(Dated: November 2, 2018)

\begin{abstract}
Motivated by recent experiments in Bose-Einstein condensed atoms that have been confined in toroidal traps, we examine the stability of persistent currents in such systems. We investigate the extent that the stability of these currents may be tunable, and the possible difficulties in their creation and detection.
\end{abstract}

PACS numbers: 05.30.Jp, 03.75.Lm, 03.75.Kk

\section{INTRODUCTION}

The recent advances in the physics of cold atoms have allowed experimentalists to engineer many of the properties of these systems. Remarkably, the experiments of Refs. 1, 2] have managed to trap atoms in toroidal traps, while in Ref. [3] persistent currents were created and observed in toroidal traps. Furthermore, in an older experiment, Ref. [4] has investigated the dissipationless flow of an obstacle in an elongated Bose-Einstein condensate below some critical velocity.

Such simple trapping geometries makes these systems very appealing mainly for two reasons, one theoretical and one technological. Theoretically these gases are ideal for testing fundamental superfluid properties (such as persistent currents, for example), and they may realize exactly-solvable one-dimensional models. Technologically, the possibility of creating persistent currents whose stability is tunable externally may lead to important, or even revolutionary applications.

Motivated by these facts, we focus in the present study on the stability of persistent currents in a quasi-one dimensional, toroidal trap. Three are the classes of problems that we consider. In the first one, we consider the situation where one manipulates the trap appropriately, in order to achieve the desired effect on the stability of currents. In the second, we investigate unavoidable complications in realistic experiments, which may affect the stability of persistent currents. The third class includes the problems associated with the detection of persistent currents.

Many aspects of the problem that we examine in our study have been investigated thoroughly in previous studies. We do not attempt to give a complete list of references, but rather we mention just few of them $[5,66,7,8,19,10,11,12,13,14,15,16,17,18,19]$.

In what follows, we first consider our model in Sec. II. Then, in Sec. III we consider a step potential along the torus and investigate the conditions that destabilize the current most easily. In Sec. IV we consider a periodic potential along the torus and examine the stability of persistent currents in such a potential. Then, in Sec. V we consider a random potential and via a statistical analysis of our results, we examine the effect of the length scale of the irregularities on the stability of the current. In Sec. VI, we investigate the effect of gravity on a tilted torus, and in Sec. VII we propose a method which allows the detection of a current. Finally, in Sec. VIII we present the conclusions of our study.

\section{MODEL}

In our model we consider one-dimensional motion of the atoms with periodic boundary conditions, which corresponds physically to the motion of the atoms in a very tight toroidal trap. Quasi-one dimensional motion is accomplished as long as the interaction energy is much smaller than the excitation energy transversely to the axis of the torus. We also assume for the atom-atom collisions the usual contact potential, $V_{\text {int }}\left(\mathbf{r}-\mathbf{r}^{\prime}\right)=U_{0} \delta\left(\mathbf{r}-\mathbf{r}^{\prime}\right)$ with $U_{0}=4 \pi \hbar^{2} a_{\mathrm{sc}} / M$. Here, $a_{\mathrm{sc}}$ is the scattering length for elastic atom-atom collisions and $M$ is the atomic mass.

The mean-field approximation that we use in this study implies that the condensate order parameter $\Psi(\theta)$ satisfies the nonlinear, Gross-Pitaevskii-like equation [20, 21],

$$
-\frac{\partial^{2} \Psi}{\partial \theta^{2}}+V(\theta) \Psi(\theta)+2 \pi \gamma|\Psi(\theta)|^{2} \Psi=\mu \Psi,
$$

where we have set $\hbar=2 M=R=1$, with $R$ being the radius of the torus. Here $\theta$ is the azimuthal angle, $V(\theta)$ is the external potential, and $\mu$ is the chemical potential. Both $V(\theta)$ and $\mu$ are measured in units of the kinetic energy $T=\hbar^{2} /\left(2 M R^{2}\right)$. The ratio between the interaction energy and the kinetic energy is equal to $\gamma=n_{0} U_{0} / T=4 N a_{\mathrm{sc}} R / S$. Here $n_{0}=N /(2 \pi R S)$ is the average atom density, $N \gg 1$ is the atom number, and $S$ is the cross section of the torus (with $R \gg \sqrt{S}$ ). As long as $\gamma \ll N^{2}$, the system is away from the TonksGirardeau limit of impenetrable bosons [22], that we do not consider in our study.

To investigate the stability of persistent currents, we 
use the time-dependent version of Eq. (1)

$$
i \hbar \frac{\partial \Psi}{\partial t}=-\frac{\partial^{2} \Psi}{\partial \theta^{2}}+V(\theta) \Psi(\theta)+2 \pi \gamma|\Psi(\theta)|^{2} \Psi .
$$

We propagate some initial state in imaginary time, making the substitution $\tau=i t$ [23], and solve the equation

$$
-\hbar \frac{\partial \Psi}{\partial \tau}=-\frac{\partial^{2} \Psi}{\partial \theta^{2}}+V(\theta) \Psi(\theta)+2 \pi \gamma|\Psi(\theta)|^{2} \Psi-\mu \Psi .
$$

More specifically, in the above equation we specify $\Psi(\theta, \tau=0)$ and look for the convergent solution that emerges for long enough times from the above timedependent equation.

The states that correspond to successive values of (the quantized) circulation may be separated by an energy barrier, provided that the interaction is strong enough 22, 24, 25]. These barriers give rise to stable persistent currents. Therefore, if one starts with the initial condition $\Psi(\theta, \tau=0)=e^{i \theta} / \sqrt{2 \pi}$ (for example) that has one unit of circulation and a uniform density distribution, if there exists an energy barrier between this state and the current-free state, then the long-time solution of Eq. (3), still has one unit of circulation, with possibly some angledependent variation in its density, depending on the form of the potential $V(\theta)$. On the other hand, in the absence of an energy barrier between the two states (which is the case for sufficiently weak, or attractive interactions) the system is energetically unstable, and it relaxes to the circulation-free state.

The criterion for the stability of circulation that we use in our study is equivalent to a condition that resembles the Landau criterion for superfluid flow 24]. More precisely, in order to have stability of the superflow, the "drift" velocity of the atoms $u$ must not exceed the speed of sound $c$. For example, for one unit of circulation that we consider here, the drift velocity is equal to $\hbar /(M R)$. Also, in the limit of weak interactions the speed of sound is given by [26] (see, e.g., Eq. (8), or Ref. [27]),

$$
c=\frac{\hbar}{2 M R}(1+\gamma)
$$

and in order for $u$ to be smaller than $c, \gamma$ has to be larger than unity, in agreement with the criterion of energetic stability. Finally, we mention that while we have restricted our study to the transition between states of circulation equal to unity and zero, similar effects occur in transitions between states of higher, successive values of the circulation.

\section{DESTABILIZATION OF PERSISTENT CURRENTS WITH A STEP POTENTIAL}

As a first application of our method, we consider a step-like potential of a fixed height (equal to unity in our units) along the torus,

$$
V(\theta)=\left\{\begin{array}{l}
1,-\pi \leq \theta \leq \theta_{0} \\
0, \theta_{0}<\theta<\pi
\end{array},\right.
$$

and investigate the critical value $\gamma_{c}$ of the coupling $\gamma$ that is necessary to maintain the stability of the current, as function of $\theta_{0}$. The form of the potential that we choose is not accidental. Among all the possible functional forms of $V(\theta)$ that we investigated with $V_{\max }-V_{\min }=1$, this is the one that requires the highest value of $\gamma_{c}$, for a static potential. Therefore under these conditions, at least within the functional forms of the potentials that we have considered, the derived value of $\gamma_{c}$ may serve as an upper bound for the critical coupling that is necessary to sustain a persistent current of circulation equal to unity.

Following the method that was described in the previous section, we plot in Fig. $1 \gamma_{c}$ versus $\theta_{0}$. As one sees in this plot, the highest value of $\gamma_{c}$ that is necessary to stabilize the current occurs for $\theta_{0} /(2 \pi) \simeq-0.18$. The position of $\theta_{0}$ decreases with increasing $V_{\max }-V_{\min }$.

For $\theta_{0} \rightarrow-\pi$, and $\theta_{0} \rightarrow \pi, \gamma_{c}$ tends to $3 / 2$, which is the critical value of $\gamma_{c}$ that corresponds to a constant $V(\theta)[20,21,27]$. For intermediate values of $\theta_{0}$ the situation becomes more interesting, as in this case the length scale of variation of $V(\theta)$ is comparable to the coherence length $\xi$ and as a result $\gamma_{c}$ is higher than 3/2. More precisely, when $\gamma$ is of order unity, the coherence length $\xi$, is $\sim R$, since $\xi / R=\gamma^{-1 / 2}$. The fact that $\xi$ is comparable with the radius of the torus, and thus comparable with the length scale of variation of $V(\theta)$, implies that the current becomes fragile. A crude guess for $\theta_{0} /(2 \pi)$ that requires the maximum value of $\gamma_{c}$ is the middle of the torus (i.e., when the step potential extends over one half of the torus), as we also discuss in the toy model that follows below. We mentioned earlier that for $V_{\max }-V_{\min }=1$ the actual value is $\simeq-0.18$, with the corresponding value of $\gamma_{c}$ being $\simeq 3.8$.

A simple toy model for this problem gives a qualitatively correct answer for $\gamma_{c}$ as function of $\theta_{0}$. Let us consider just the states $\Phi_{0}(\theta)=1 / \sqrt{2 \pi}$ and $\Phi_{1}(\theta)=$ $e^{i \theta} / \sqrt{2 \pi}$ in the order parameter, in the form

$$
\Psi_{\text {trial }}(\theta)=\sqrt{1-l} \Phi_{0}(\theta)+e^{i \lambda} \sqrt{l} \Phi_{1}(\theta),
$$

where $\lambda$ is some phase factor. The value of $\lambda$ is determined from the minimization of the potential energy, which turns out to be $\lambda=-\left(\theta_{0}+\pi\right) / 2$. Clearly the above state has an expectation value of the angular momentum per particle equal to $l$. The corresponding density is

$$
n_{\text {trial }}(\theta)=\frac{1}{2 \pi}\left[1+2 \sqrt{l(1-l)} \sin \left(\theta-\theta_{0} / 2\right)\right],
$$

and also the (minimized) energy per particle is, for the step potential of Eq. (5),

$$
E-\frac{\gamma}{2}-\frac{\theta_{0}+\pi}{2 \pi}=(1+\gamma) l-\gamma l^{2}-\sqrt{l(1-l)} \frac{2}{\pi} \cos \left(\frac{\theta_{0}}{2}\right) .
$$

From the above dispersion relation we find the critical value $\gamma_{c}$ that gives rise to a local minimum close to $l=1$. The specific (highly truncated) order parameter gives $\gamma_{c}=1$ for a constant potential (i.e., for $\theta_{0}= \pm \pi$ ), while 


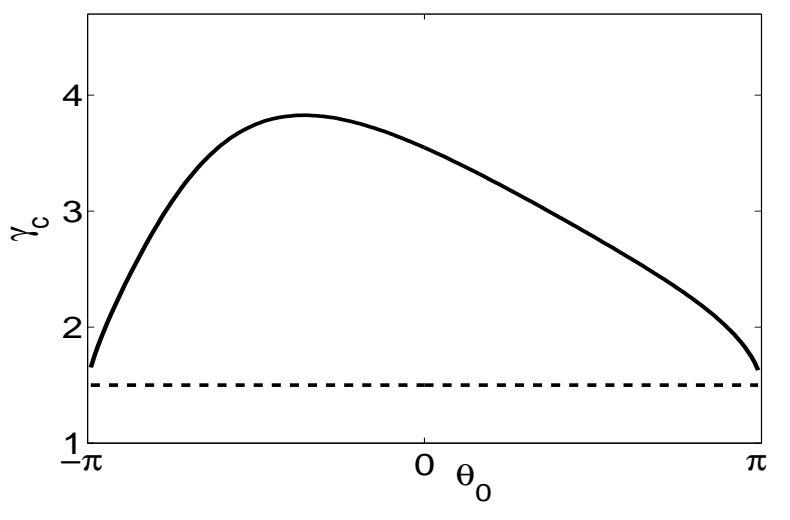

FIG. 1: The critical value of the coupling constant $\gamma_{c}$ versus the location of the step $\theta_{0}$, in a step potential of the form of $V(\theta)$ defined in Eq. (5). The value of $\gamma_{c}=3 / 2$ for the uniform case (dashed line) is also shown as a reference.

$\gamma_{c}$ has a maximum for $\theta_{0}=0$, equal to $\approx 2.295$. In addition, $\gamma_{c}\left(\theta_{0}\right)$ is symmetric around $\theta_{0}=0$. In the limit of weak disorder and weak coupling, where the actual order parameter is closer to $\Psi_{\text {trial }}(\theta)$, the density profile of the cloud is sinusoidal, and the step potential just determines the position of the maximum/minimum of the density, as one can see in Eq. (77). As a result, $\gamma_{c}\left(-\theta_{0}\right)=\gamma_{c}\left(\theta_{0}\right)$. For higher couplings and higher values of $V_{\max }-V_{\min }$, the density of the cloud gets distorted from the sinusoidal form, as it localizes more in the region of lower potential energy. The function $\gamma_{c}\left(\theta_{0}\right)$ becomes then asymmetric with respect to $\theta_{0}=0$, as shown in Fig. 1 (which shows the full numerical calculation.)

\section{STABILITY OF PERSISTENT CURRENTS IN THE PRESENCE OF A PERIODIC POTENTIAL}

Another interesting question is the stability of persistent currents in the presence of a periodic potential that acts along the torus. Experiments with periodic potentials created by optical lattices have already been performed [28]. Numerous theoretical studies have examined this problem, too. This is a very interesting problem, since the presence of a periodic external potential, combined with the effect of the interactions give rise to novel states. For example, we refer to Refs. [29, 30, 31, 32, 33] for studies of the Gross-Pitaevskii equation in the presence of a periodic potential, for calculations of the band structure, and for the study of the superfluid properties of these systems.

In our study we consider a sinusoidal external potential of the form

$$
V(\theta)=\cos (m \theta),
$$

where $m=1,2,3, \ldots$, as the condition $V(\theta+2 \pi)=V(\theta)$ requires. This potential has a period equal to $2 \pi / \mathrm{m}$. Again, we examine the stability of a current with one unit

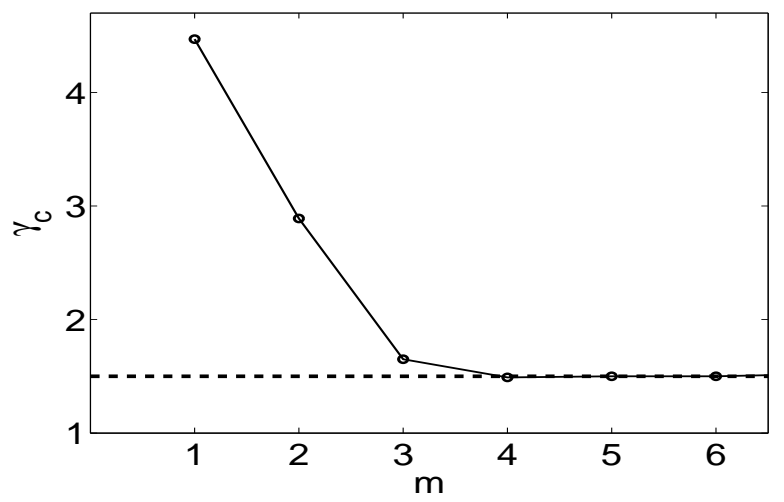

FIG. 2: The critical value of $\gamma_{c}$ versus the parameter $m$ (dots), for the periodic potential of Eq. (9). The solid line is a guide for the eye. The value of $\gamma_{c}=3 / 2$ for the uniform case (dashed line) is also shown as a reference.

of circulation, starting with $\Psi(\theta, \tau=0)=e^{i \theta} / \sqrt{2 \pi}$ and finding the critical value of $\gamma$ that gives rise to a stable current, for various values of $m=1, \ldots, 6$. The results of this calculation are shown in Fig. 2. As $m$ increases, the value of $\gamma_{c}$ that is required to give rise to a stable current decreases.

For $m=1$, the potential is qualitatively similar to a step potential examined in Sec.III, but it is smoother (which decreases $\gamma_{c}$ ) and has a larger $V_{\max }-V_{\min }=2$ (which increases $\gamma_{c}$ ). For a step potential with $V_{\max }-$ $V_{\min }=2$, that is located in the middle of the torus $\left(\theta_{0}=0\right)$, one finds $\gamma_{c} \simeq 4.8$, in rough agreement with the maximum value of $\gamma_{c}$ shown in Fig. 2. Furthermore, as $m$ increases, eventually the potential becomes homogeneous (on the length scale of variation of the density), in which case $\gamma_{c}=3 / 2[20,21$, 27]. This is clearly seen in Fig. 2. The crossover region to the homogeneous case is accomplished when the "lattice constant" $\pi / m$, associated with the periodic potential $V(\theta)$, is $\sim \xi / R=\gamma_{c}^{-1 / 2}$. The above equation implies that $m \sim \pi \gamma_{c}^{1 / 2}=\pi(3 / 2)^{1 / 2} \approx 3.9$, in (rough) agreement with our result shown in Fig. 2.

\section{STABILIZATION OF A CURRENT IN THE PRESENCE OF A RANDOM POTENTIAL}

In a previous study [27], we investigated the value of $\gamma_{c}$ that is necessary to stabilize a current in the presence of a piecewise constant potential, with a randomly chosen amplitude. Here we present data that we have collected from a statistical analysis of our simulations, where we examine $\gamma_{c}$ as function of the number of steps that we choose in $V(\theta)$.

More specifically, we compute the average value of $\gamma_{c}$, as well as the standard deviation $\sigma\left(\gamma_{c}\right)$, for 1000 different random step potentials, for which there are $s$ steps, with a width $(2 \pi / s)$. The value of the potential within each step is drawn uniformly from the interval $[-1,1]$. Figure 


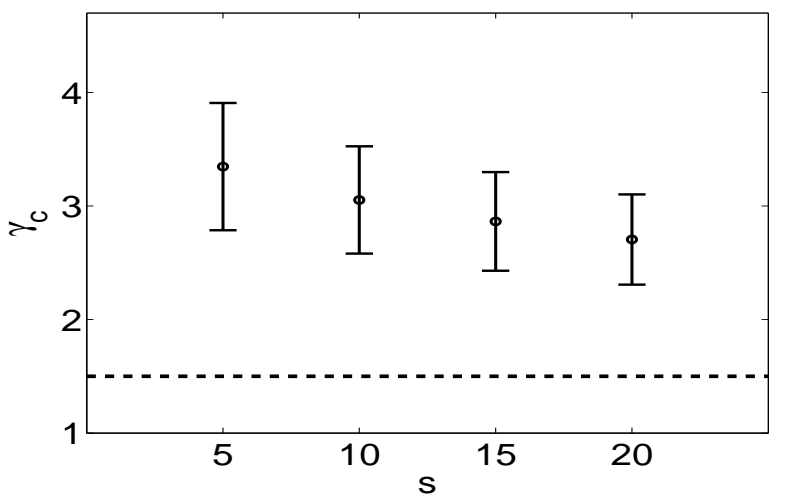

FIG. 3: The average value of the critical coupling $\left\langle\gamma_{c}\right\rangle$ (dots), as well as the standard deviation (bars), versus the number of steps $s$ in a randomly-chosen potential. These results are derived from 1000 different random potentials for each number of steps $s=5,10,15,20$. The value of $\gamma_{c}=3 / 2$ for the uniform case (dashed line) is also shown as a reference.

3 shows the result of these calculations. We observe that $\left\langle\gamma_{c}\right\rangle$ is a decreasing function of $s$.

As in the case of a step potential, one may argue that as $s$ increases, the length scale of variation of the random potential becomes increasingly smaller than the coherence length, and eventually one recovers the result $\gamma_{c}=3 / 2$ of the homogeneous torus, when $V(\theta)$ is constant. On the other hand, as $s$ decreases, eventually the two length scales become comparable and the random potential destabilizes the current more easily, requiring a higher value of $\gamma_{c}$ for the current to become stable.

\section{EFFECT OF GRAVITY ON THE STABILITY OF PERSISTENT CURRENTS}

One question that needs to be investigated within the problems that we examine here is the effect of gravity on the stability of persistent currents. Already in other experiments with cold atoms, gravity has played an important role, see e.g. [34]. In real life, the torus cannot be perfectly horizontal, and as a result, there is an angledependent potential that acts on the atoms due to the gravitational force. This potential is

$$
V(\theta)=(M g R \sin \delta) \cos \theta,
$$

where $g$ is the acceleration of gravity and $\delta$ is the angle between the plane of the torus and the horizontal plane.

Figure 4 shows $\gamma_{c}$ as function of $\log _{10}(\delta)$, for three values of $R=1 \mathrm{~mm}$ (left), $0.1 \mathrm{~mm}$ (middle), and 0.01 $\mathrm{mm}$ (right). As the angle $\delta$ increases for a given $R, \gamma_{c}$ increases too, since the effect of the gravitational field gets more important. For the same reason, for a fixed angle $\delta$, as the radius of the torus $R$ increases, the critical value of $\gamma_{c}$ increases, too. For the small values of the angle $\delta$ that we have considered, $\sin \delta \approx \delta$, and therefore the shape of the three different curves that are shown

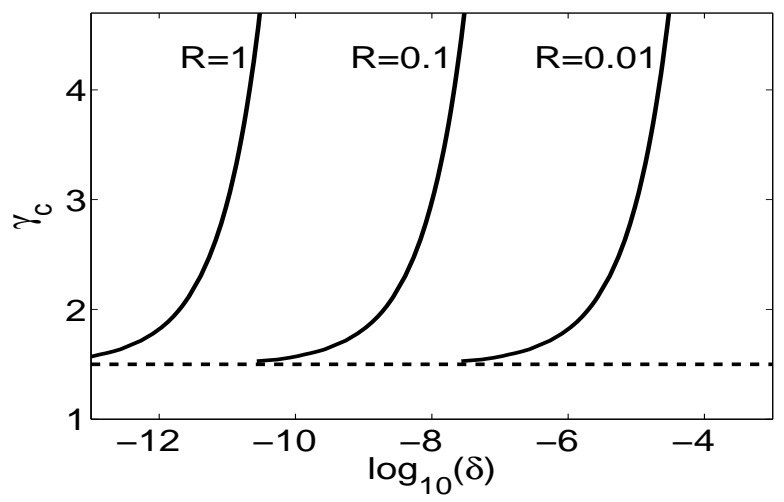

FIG. 4: The critical value of $\gamma_{c}$ versus $\log _{10}(\delta)$, for $R=1 \mathrm{~mm}$ (left), $0.1 \mathrm{~mm}$ (middle), and $0.01 \mathrm{~mm}$ (right). The value of $\gamma_{c}=3 / 2$ for the uniform case (dashed line) is also shown as a reference.

(which correspond to the three different values of $R$ ), are almost identical, as they can be obtained approximately by a shift in $\delta$, which is $\propto R^{-3}$.

It is instructive to get an estimate for the energy scale $M g R$ in Eq. (10). Considering Rubidium atoms, for example, and for a radius of the torus $R=0.1 \mathrm{~mm}, M g R$ is on the order of $\mu \mathrm{K}$, which is a rather large energy scale. Therefore, in order for the effect of gravity to be unimportant, the torus has to be tilted slightly, in which case $\sin \delta \ll 1$.

\section{DETECTION OF PERSISTENT CURRENTS}

A serious issue in these experiments is to be able to know whether there is circulation in the gas, or not. This question can be resolved with use of interference techniques [35], however it would be convenient to have also a way to measure the circulation using other methods.

It is natural to think of the single-particle density distribution $n(\theta)=|\Psi(\theta)|^{2}$ as a possible way to determine the value of the circulation. Any constant potential $V(\theta)$ results in a homogeneous density distribution, as the order parameter is $e^{i \kappa \theta} / \sqrt{2 \pi}$, for any value of the circulation $\kappa(2 \pi \hbar / M)$. On the other hand, if one uses a probe potential $V(\theta)$ that is spatially-dependent, the density distribution $n(\theta)$ does depend on the value of $\kappa$. Therefore, measuring the density in the presence of some spatially-dependent potential $V(\theta)$ may allow us to determine the value of the circulation.

We thus consider such a probe potential in the form of a Gaussian unity dimple (which may be realized via some laser beam) with (rms) width $w_{d}$

$$
V(\theta)=-\exp \left(-\theta^{2} / 2 w_{d}^{2}\right)
$$

Figure 5 shows the density distribution $n(\theta)$ in the presence of $V(\theta)$ of Eq. (11), with $w_{d}=0.2$ for the states with zero circulation $(\kappa=0)$, and unit circulation $(\kappa=1)$. 


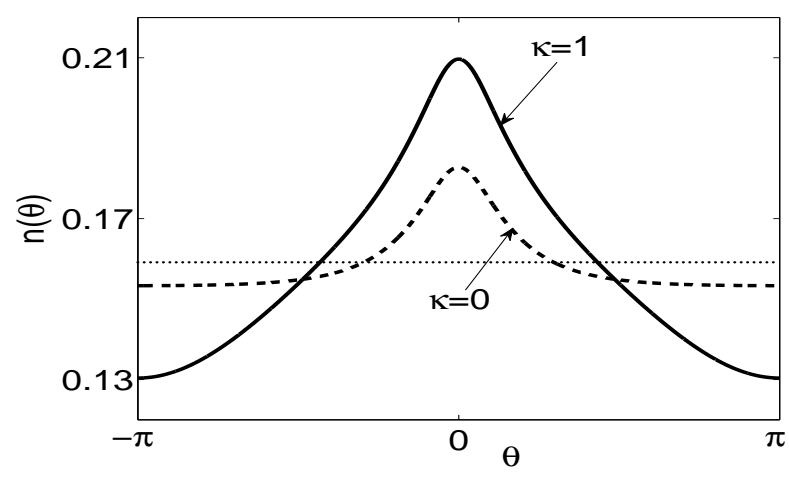

FIG. 5: The (normalized) density distribution $n(\theta)$ of the state of lowest energy, in the presence of $V(\theta)$ given by Eq. (11), for zero circulation, $\kappa=0$ (dashed curve), and unit circulation $\kappa=1$ (solid curve), for a coupling strength $\gamma=2.2$. The horizontal dotted line shows the density of the uniform state, $n(\theta)=1 / 2 \pi$. All densities are measured in units of $N /(R S)$.

The chosen value of the coupling is larger than the critical one, $\gamma=2.2>\gamma_{c} \approx 2.1$. The state with $\kappa=1$ has a higher kinetic energy, and as a result the classicallyforbidden region is more narrow, resulting in a wider density distribution around the minimum of the probe potential $V(\theta)$. Ideally one would like the density profiles corresponding to different values of the circulation to be as different as possible, even in the limit of a very weak probe potential $|V(\theta)| \ll 1$.

To quantify the difference between the two density distributions illustrated in Fig. 5, one may introduce the difference between the highest and the lowest value of the density in each case, $\Delta n=n_{\max }-n_{\min }=n(\theta=$ $0)-n(\theta= \pm \pi)$. Remarkably, this quantity differs by more than a factor of two $(\approx 2.7)$ between the two density profiles that we consider in the specific example.
The above results indicate that a possible way to measure the circulation (non-destructively), would be to turn on the probe potential adiabatically, measure the density and extract the value of $\kappa$ (non-destructively), and finally turn off the probe adiabatically again.

\section{SUMMARY AND CONCLUSIONS}

In this study we examined the behavior of atoms that circulate in a tight toroidal trap. Manipulation of the trapping potential along the torus may provide various ways to control the stability of the current. The form of the potentials that we considered include a step potential, a sinusoidal potential, a random potential, and a gravitational potential. We calculated the value of the critical coupling that gives rise to stable persistent currents. In addition, we suggested a method that allows the detection of circulation. This method involves a probe, spatially-dependent potential, which, for different values of the circulation gives a different density variation. This method requires only a rough measurement of the density profile, which may be realizable non-destructively [36].

Our results indicate that the remarkable progress on the manipulation of the trapping potential 37], and more generally the developments in the physics of cold atoms may allow us to engineer such mesoscopic systems that support persistent currents according to our will. Such systems may therefore serve as "superconducting switches" with possible important technological applications.

\section{Acknowledgments}

We thank Jozsef Fortagh and Andrew Sykes for useful discussions.
[1] S. Gupta, K. W. Murch, K. L. Moore, T. P. Purdy, and D. M. Stamper-Kurn, Phys. Rev. Lett. 95, 143201 (2005).

[2] S. E. Olson, M. L. Terraciano, M. Bashkansky, F. K. Fatemi, Phys. Rev. A 76, 061404(R) (2007).

[3] C. Ryu, M. F. Andersen, P. Cladé, V. Natarajan, K. Helmerson, and W. D. Phillips, Phys. Rev. Lett. 99, 260401 (2007).

[4] C. Raman, M. Köhl, R. Onofrio, D. S. Durfee, C. E. Kuklewicz, Z. Hadzibabic, and W. Ketterle, Phys. Rev. Lett. 83, 2502 (1999).

[5] M. Ma, B. I. Halperin, and P. A. Lee, Phys. Rev. B 34, 3136 (1986).

[6] M. P. A. Fisher, P. B. Weichman, G. Grinstein, and D. S. Fisher, Phys. Rev. B 40, 546 (1989).

[7] K. Huang and H.-F. Meng, Phys. Rev. Lett. 69, 644 (1992).

[8] S. Giorgini, L. Pitaevskii, and S. Stringari, Phys. Rev. B 49, 12938 (1994).
[9] V. Hakim, Phys. Rev. E 55, 2835 (1997).

[10] N. Pavloff, Phys. Rev. A 66, 013610 (2002).

[11] G. E. Astrakharchik, J. Boronat, J. Casulleras, and S. Giorgini, Phys. Rev. A 66, 023603 (2002).

[12] M. Kobayashi and M. Tsubota, Phys. Rev. B 66, 174516 (2002).

[13] P. Navez, A. Pelster, and R. Graham, Appl. Phys. B 86, 395 (2007).

[14] L. Sanchez-Palencia, Phys. Rev. A 74, 053625 (2006)

[15] J. E. Lye, L. Fallani, M. Modugno, D. S. Wiersma, C. Fort, and M. Inguscio, Phys. Rev. Lett. 95, 070401 (2005).

[16] C. Fort, L. Fallani, V. Guarrera, J. E. Lye, M. Modugno, D. S. Wiersma, and M. Inguscio, Phys. Rev. Lett. 95, 170410 (2005).

[17] D. Clément, A. F. Varón, M. Hugbart, J. A. Retter, P. Bouyer, L. Sanchez-Palencia, D. M. Gangardt, G. V. Shlyapnikov, and A. Aspect, Phys. Rev. Lett. 95, 170409 
(2005).

[18] D. Clément A. F. Varon, J. A. Retter, L. SanchezPalencia, A. Aspect, and P. Bouyer, New Journal of Physics 8, 165 (2006); P. Lugan, D. Clément, P. Bouyer, A. Aspect, M. Lewenstein, and L. Sanchez-Palencia, Phys. Rev. Lett. 98, 170403 (2007).

[19] T. Paul, P. Schlagheck, P. Leboeuf and N. Pavloff, Phys. Rev. Lett. 98, 210602 (2007).

[20] R. Kanamoto, H. Saito, and M. Ueda, Phys. Rev. A 68, 043619 (2003).

[21] G. M. Kavoulakis, Phys. Rev. A 69, 023613 (2004).

[22] G. M. Kavoulakis, Y. Yu, M. Ögren, and S. M. Reimann, Europhys. Lett. 76, 215 (2006).

[23] S. A. Chin and E. Krotscheck, Phys. Rev. E 72, 036705 (2005).

[24] F. Bloch, Phys. Rev. A 7, 2187 (1973).

[25] A. J. Leggett, Rev. Mod. Phys. 73, 307 (2001).

[26] This result implies that $M c^{2}=\hbar^{2} /\left(4 M R^{2}\right)+$ $2 \hbar^{2} N a_{\mathrm{sc}} /(M R S)+\mathcal{O}\left(\gamma^{2}\right)$. In the limit $N \rightarrow \infty$ and $R \rightarrow \infty$, with $N / R$ finite, $M c^{2}=2 \hbar^{2} N a_{\text {sc }} /(M R S)$, in agreement with the formula $M c^{2}=n_{0} U_{0}$, which is known for a homogeneous gas of density $n_{0}=N /(2 \pi R S)$.

[27] M. Ögren and G. M. Kavoulakis, Journal of Low Temp. Phys. 149, 176 (2007).

[28] B. P. Anderson and M. A. Kasevich, Science 282, 1686
(1998); S. Burger, F. S. Cataliotti, C. Fort, F. Minardi, M. Inguscio, M. L. Chiofalo, and M. P. Tosi, Phys. Rev. Lett. 86, 4447 (2001); F. S. Cataliotti, S. Burger, C. Fort, P. Maddaloni, F. Minardi, M. Inguscio, M. L. Chiofalo, and M. P. Tosi, Science 293, 843 (2001); O. Morsch, J. H. Müller, M. Cristiani, D. Ciampini, and E. Arimondo, Phys. Rev. Lett. 87, 140402 (2001).

[29] B. Wu and Q. Niu, Phys. Rev. A 61, 023402 (2000).

[30] J. C. Bronski, L. D. Carr, B. Deconinck, and J. N. Kutz, Phys. Rev. Lett. 86, 1402 (2001).

[31] D. Diakonov, L. M. Jensen, C. J. Pethick, and H. Smith, Phys. Rev. A 66, 013604 (2002).

[32] M. Machholm, C. J. Pethick, and H. Smith, Phys. Rev. A 67, 053613 (2003).

[33] B. Wu and Q. Niu, New Journal of Physics 5, 104 (2003).

[34] M.-O. Mewes, M. R. Andrews, D. M. Kurn, D. S. Durfee, C. G. Townsend, and W. Ketterle, Phys. Rev. Lett. 78, 582 (1997).

[35] F. Chevy, K. W. Madison, V. Bretin, and J. Dalibard, Phys. Rev. A 64, 031601(R) (2001).

[36] F. Brennecke, T. Donner, S. Ritter, T. Bourdel, M. Köhl, and T. Esslinger, Nature 450, 268 (2007).

[37] S. K. Schnelle, E. D. van Ooijen, M. J. Davis, N. R. Heckenberg, and H. Rubinsztein-Dunlop, e-print arXiv:0801.2791v1. 